\title{
Effect of Irreversible Atomic Relaxation on Resonance Fluorescence, Absorption, and Stimulated Emission
}

\author{
Gian Paolo Beretta ${ }^{1}$ \\ Received January 11, 1985
}

\begin{abstract}
Even for a single isolated constituent of matter, a recent generalization of quantum mechanics, called quantum thermodynamics, postulates the existence of new nonmechanical individual states, not contemplated within conventional quantum mechanics, for which the time evolution is governed by a novel nonlinear equation of motion, which entails an irreversible, energy-preserving internal redistribution mechanism of relaxation towards stable equilibrium. For a single two-level atom interacting with the quantum electromagnetic field, we show that such irreversible internal redistribution mechanism entails interesting corrections to the conventional quantum electrodynamic predictions on absorption, resonance fluorescence, and stimulated emission. For a two-level atom driven near resonance by a nearly monochromatic laser beam, we estimate the corrections implied on the spectral distribution of resonance fluorescence and on the absorption and stimulated emission line shape. We submit that our predictions call for further high-resolution studies of atom-field interactions. For example, the value or a lower bound to the value of the only unknown constant of the theory, namely, the internal redistribution time constant, can only be established by a quantitative experimental study.
\end{abstract}

\section{INTRODUCTION}

The purpose of this article is to study the interaction between a two-level atom and the quantum electromagnetic field, within the framework of a novel generalized quantum dynamical theory which contains conventional quantum theory as a special case.

The spectral distribution of the fluorescence resulting from a nearly resonant interaction has been first calculated by Mollow (1969) and later confirmed by several more rigorous treatments (Ackerhalt and Eberly, 1974; Kimble and Mandel, 1975; 1977; Cohen-Tannoudji and Reynaud, 1977;

\footnotetext{
${ }^{1}$ Massachusetts Institute of Technology, Room 3-339, Cambridge, Massachusetts 02139, and Politecnico di Milano, 20133 Milano, Italy.
} 
Mollow, 1972; 1975; 1981) all within the framework of conventional quantum electrodynamics. Recent experimental studies (Schuda et al., 1974; Wu et al., 1975; Hartig et al., 1976; Grove et al., 1977; Wu et al., 1977; Citron, et al., 1977; Ezekiel and Wu, 1978; Cresser et al., 1982) of the phenomenon have verified the quantum electrodynamic predictions qualitatively. Quantitative comparisons between theory and experiments are satisfactory but still not conclusive.

In an attempt to resolve the long-standing dilemma on the nature of entropy and irreversibility, ${ }^{2}$ we have recently proposed a novel nonstatistical generalized quantum dynamical theory (Beretta et al., 1984; Beretta et al., 1985; Beretta, in press) based on two fundamental hypotheses new to physics. We review the background motivation and the basic premises of the new theory, that we call quantum thermodynamics, in Section 2. Inherent to each elementary constituent of matter, including a single isolated twolevel atom, quantum thermodynamics avers the existence of an irreversible energy-preserving internal redistribution mechanism not contemplated by conventional quantum theory.

The main question addressed in this paper is whether the new theory entails experimentally verifiable predictions different from those of conventional quantum theory. To this end, we estimate the corrections implied by our nonconventional generalized quantum dynamics onto the conventional quantum electrodynamic predictions on the spectral distribution of resonance fluorescence and the absorption and stimulated emission line shape.

Our objective is not to give a complete and fully rigorous treatment of atom-field interactions within quantum thermodynamics. Rather, it is to present a preliminary study that we hope will motivate higher resolution experimental investigations and more rigorous theoretical studies of such corrections.

The article is organized as follows. Section 2 reviews background, motivation and premises of quantum thermodynamics. Section 3 presents the nonlinear equation of motion that we postulate for a single isolated two-level system. Section 4 briefly reviews the accepted quantum electrodynamic equations for a two-level atom interacting with the electromagnetic field under the standard approximations. Section 5 presents our approximate corrections to the quantum electrodynamic equations. The equations are solved in the steady-state long-time limit by introducing a useful shorthand notation. Sections 6 and 7 discuss important consequences of our postulates on the spectral distribution of resonance fluorescence and the absorption and stimulated emission line shape.

${ }^{2}$ For a recent review, see Park and Simmons (1983). 


\section{QUANTUM THERMODYNAMICS}

Quantum thermodynamics is a generalization of quantum mechanics designed to encompass within a single nonstatistical theory all the results of mechanics and thermodynamics. It is based on the following two fundamental hypotheses that are new to physics and demand both theoretical and experimental scrutiny.

The first hypothesis (Hatsopoulos and Gyftopoulos, 1976a-d) is that, in addition to the individual quantum states conceived of within conventional quantum theory and represented by indempotent state operators, a single strictly isolated system admits also of individual quantum states that must be represented by nonindempotent state operators. A state operator $\rho$ is a linear, self-adjoint, nonnegative-definite, unit-trace operator on the Hilbert space of the isolated system. Every idempotent state operator $(\rho=$ $\left.\rho^{2}=P_{\psi}\right)$ is a projector onto the one-dimensional span of a vector $\psi$ in the Hilbert space, called the quantum mechanical state vector of the isolated system. For an individual physical state of an isolated (noninteracting and uncorrelated) system, i.e., for a strictly homogeneous (von Neumann) ensemble of such systems, conventional quantum mechanics conceives only of the description in terms of a quantum mechanical state vector or, equivalently, an idempotent state operator. Nonidempotent (statistical or density) operators are used within conventional quantum theory only to describe nonindividual conditions, such as the reduced state of a system correlated to other systems or the index of statistics from a heterogeneous (von Neumann) ensemble of identical systems. These conditions are excluded when we refer to an individual state of an isolated (uncorrelated) system. Quantum thermodynamics is concerned exclusively with physical conditions that within conventional quantum theory would be described by idempotent (pure) statistical or density operators. For a system in such conditions, it postulates that the individual states must be described in terms of state operators that need not be idempotent. In terms of ensembles, the essence of the Hatsopoulos-Gyftopoulos hypothesis is that we conceive of a pure or homogeneous ensemble (each member system of which is exactly in the same individual physical state as all the other member systems) that in our generalized quantum theory is represented by a state operator, not necessarily idempotent. The state operator (even if nonidempotent) represents the individual state of a single system, not the index of statistics from a generally heterogeneous ensemble. For this reason, we say that our theory is nonstatistical. Indeed, the only uncertainties with which it is concerned are those inherent in the individual quantum state. The second hypothesis is that state operators evolve in time according to a nonlinear equation of motion (Beretta, 1981) which for indempotent state operators 
reduces to the Schroedinger equation of motion of conventional quantum dynamics, but for most nonidempotent individual state operators describes a constant energy, entropy increasing, irreversible relaxation towards a stable equilibrium state. ${ }^{3}$

Quantum thermodynamics has been developed in answer to the following two fundamental questions: (1) Can entropy and the second law of thermodynamics be conceived of exclusively as manifestations of microscopic quantum kinematic effects of the same nature but more general than those entailed by the Heisenberg uncertainty principle? (2) Can irreversibility be conceived of exclusively as a microscopic quantum dynamical effect that must be described by a proper generalization of the Schroedinger equation of motion? Quantum thermodynamics yields an affirmative answer to both questions. It encompasses quantum mechanics and equilibrium thermodynamics as special cases, it includes a new general microscopic nonstatistical description of nonequilibrium states, and it entails a unique deterministic description of irreversible but conservative relaxation towards equilibrium, based on the nonlinear irreversible equation of motion proposed by the author.

The question that remains open is whether quantum thermodynamics entails experimentally verifiable predictions that are different from the predictions of conventional quantum theory. The aim of this paper is to show that indeed the new theory implies corrections to the conventional results. However, the magnitude of these corrections depends on a time constant the value of which can only be determined experimentally. We hope that our results will motivate experimental studies to verify the quantum thermodynamic corrections or at least determine a bound to the value of the internal redistribution time constant.

To estimate the corrections implied by quantum thermodynamics onto the conventional quantum electrodynamic theory of resonance fluorescence, absorption, and stimulated emission, we proceed as follows. In Section 3, we write our equation of motion for a single isolated two-level atom with an arbitrary Hamiltonian operator. The equation consists of two terms: the first describes the conventional Hamiltonian contribution to the dynamics of the state operator, the second describes the postulated irreversible, conservative, inherent redistribution mechanism. In Section 4 , for a system composed of a two-level atom and a nearly resonant electromagnetic field, we review the accepted quantum electrodynamic treatment (Ackerhalt and

\footnotetext{
${ }^{3}$ The form of the new equation for a single elementary constituent of matter, i.e., a single particle or a single quantum field, and detailed proofs of its most important general properties are presented in Beretta et al., (1984). The form of the equation of motion for a single isolated two-level system, that we will adopt here to describe the internal dynamics of a two-level atom, is further discussed in Beretta (1985).
} 
Eberly, 1974; Kimble and Mandel, 1975; 1977; Cohen-Tannoudji and Reynaud, 1977; Mollow, 1972; 1975; 1981) of the conventional Hamiltonian contribution to the evolution of the reduced state operator of the two-level atom. In Section 5, to the conventional treatment we add the non-Hamiltonian nonlinear term of the new equation of motion which represents the effect of the postulated additional relaxation mechanism inherent to the two-level atom.

As a result, we find estimates for the corrections entailed by our postulates onto the absorption spectrum, the spectral density of resonance fluorescence, and the absorption and stimulated emission line shape. All the results are in terms of an unknown constant, namely, the atomic redistribution time constant. This constant must be estimated empirically, it cannot be "derived" from other theories because no other theory contemplates our nonindempotent individual states and our nonlinear equation of motion.

\section{NONLINEAR EQUATION OF MOTION FOR A SINGLE ISOLATED TWO-LEVEL SYSTEM}

We postulate that the individual state operator $\rho$ of a single isolated two-level system with Hamiltonian operator $H$, evolves in time according to the equation (Beretta, 1985)

$$
\frac{d \rho}{d t}+\frac{i}{\hbar}[H, \rho]=\left\{\begin{array}{lll}
\rho-\frac{1}{\tau} \frac{\left|\begin{array}{ccc}
\rho \ln \rho & \rho & \frac{1}{2}\{\rho, H\} \\
\operatorname{Tr} \rho \ln \rho & 1 & \operatorname{Tr} \rho H \\
\operatorname{Tr} \rho \ln \rho & \operatorname{Tr} \rho H & \operatorname{Tr} \rho H^{2}
\end{array}\right|}{\operatorname{Tr} \rho H^{2}-(\operatorname{Tr} \rho H)^{2}}, & \text { if } \rho^{2} \neq \rho \\
0, & \text { if } \rho^{2}=\rho
\end{array}\right.
$$

where $\tau$ is an inherent relaxation time constant of the system. Determining the value or bounds to the value of $\tau$ is an open experimental problem.

It is useful to introduce the three spin operators $R_{1}, R_{2}$, and $R_{3}$ which obey the commutation rule $\left[R_{j}, R_{m}\right]=i \varepsilon_{j m n} R_{n}$ and may be expressed in terms of the lowering and raising operators $b=R_{1}-i R_{2}$ and $b^{\dagger}=R_{1}+i R_{2}{ }^{4}$ The state operator $\rho(t)$ may then be represented as

$$
\begin{aligned}
\rho(t) & =\frac{1}{2} I+r_{1}(t) R_{1}+r_{2}(t) R_{2}+r_{3}(t) R_{3}=\frac{1}{2} I+\mathbf{r}(t) \cdot \mathbf{R} \\
& =\frac{1}{2} I+\alpha^{*}(t) b^{\dagger}+\alpha(t) b+r_{3}(t) R_{3}
\end{aligned}
$$

${ }^{4}$ Other useful relations are $R_{j}=\sigma_{j} / 2$, where $\sigma_{1}, \sigma_{2}$, and $\sigma_{3}$ are the Pauli spin operators; $R_{1}=\left(b+b^{\dagger}\right) / 2, \quad R_{2}=i\left(b-b^{\dagger}\right) / 2, \quad R_{3}=\left[b^{\dagger}, b\right] / 2=b^{\dagger} b-I / 2=I / 2-b b^{\dagger} ;\left\{b^{\dagger}, b\right\}=I ; \quad b^{2}=$ $b^{\dagger 2}=0 ; b R_{3}=b / 2, R_{3} b=-b / 2, R_{3} b^{\dagger}=b^{\dagger} / 2, b^{\dagger} R_{3}=-b^{\dagger} / 2$. 
The nonnegativity of state operators implies that $r(t)=|\mathbf{r}(t)| \leq 1$. A general Hamiltonian operator corresponding to the energy relative to a point midway between the two energy levels may be represented as

$$
\begin{aligned}
H & =\hbar \Omega_{0}\left(\Lambda_{1} R_{1}+\Lambda_{2} R_{2}+\Lambda_{3} R_{3}\right)=\hbar \Omega_{0} \Lambda \cdot \mathbf{R} \\
& =-\frac{1}{2} \hbar \Omega\left(\varepsilon b^{\dagger}+\varepsilon^{*} b\right)+\hbar \omega_{0} R_{3}
\end{aligned}
$$

where $\omega_{0}=\Omega_{0} \Lambda_{3}$ is the transition frequency between the two levels, $\Lambda=$ $\left(\Lambda_{1}, \Lambda_{2}, \Lambda_{3}\right)$ is a 3-vector with $\Lambda=|\Lambda|=1, \varepsilon$ is a complex scalar with $\varepsilon^{*} \varepsilon=1$, and $\Omega_{0}^{2}=\Omega^{2}+\omega_{0}^{2}$.

Using Relations 2 and 3 for $\mathbf{r} \neq \pm \boldsymbol{\Lambda},{ }^{5}$ equation (1) reduces to the following two equivalent forms ${ }^{6}$

$$
\frac{d \mathbf{r}}{d t}-\Omega_{0} \mathbf{\Lambda} \times \mathbf{r}=-\frac{1}{\tau} K(\mathbf{r})[\mathbf{r}-(\mathbf{\Lambda} \cdot \mathbf{r}) \mathbf{\Lambda}]
$$

or

$$
\begin{gathered}
\frac{d \alpha}{d t}+i \omega_{0} \alpha+\frac{i \Omega \varepsilon r_{3}}{2}=-\frac{K\left(\alpha, r_{3}\right)}{\tau \Omega_{0}^{2}}\left[\omega_{0}^{2} \alpha+\frac{\omega_{0} \Omega \varepsilon r_{3}}{2}+\frac{\Omega^{2} \varepsilon\left(\varepsilon^{*} \alpha-\varepsilon \alpha^{*}\right)}{2}\right] \\
\frac{d r_{3}}{d t}+i \Omega\left(\varepsilon^{*} \alpha-\varepsilon \alpha^{*}\right)=-\frac{K\left(\alpha, r_{3}\right)}{\tau \Omega_{0}^{2}}\left[\Omega^{2} r_{3}+\omega_{0} \Omega\left(\varepsilon^{*} \alpha+\varepsilon \alpha^{*}\right)\right]
\end{gathered}
$$

where

$$
K(\mathbf{r})=\frac{f(r)}{1-(\boldsymbol{\Lambda} \cdot \mathbf{r})^{2}}=K\left(\alpha, r_{3}\right)=\frac{f(r)}{1-\left[\omega_{0} r_{3}-\Omega\left(\varepsilon \alpha+\varepsilon^{*} \alpha^{*}\right)\right]^{2} / \Omega_{0}^{2}}
$$

$r=|\mathbf{r}|$, and

$$
f(r)= \begin{cases}1, & \text { if } r=0 \\ \frac{1-r^{2}}{2 r} \ln \frac{1+r}{1-r}, & \text { if } 0<r<1 \\ 0, & \text { if } r=1\end{cases}
$$

${ }^{5}$ If $\mathbf{r}= \pm \boldsymbol{\Lambda}, \rho_{ \pm}=\rho_{ \pm}^{2}=\boldsymbol{P}_{\psi_{ \pm}}$, where $\psi_{ \pm}$are the eigenvectors of the Hamiltonian. Then equation (1) yields $d \mathbf{r} / d t=0$, i.e., such two states are equilibrium states.

${ }^{6}$ Details of this derivation are given in Beretta (1985) where we prove that equation (1) is a well-defined evolution equation admitting unique solutions through every initial state operator. For example, we show that equation (1) preserves the nonnegativity of all its solutions for $-\infty<t<+\infty$. The solution through an initial state $\rho(0) \neq \rho^{2}(0)$ is a spiral on a constant mean energy plane which originates (as $t \rightarrow-\infty$ ) near (but not from) the periodic (Schrödinger) quantum mechanic unitary boundary solution with $\rho^{2}=\rho$ and tends (as $t \rightarrow+\infty)$ towards a stable equilibrium state. 


\section{QUANTUM ELECTRODYNAMIC REDUCED EQUATIONS OF MOTION}

We now briefly review the results of the quantum electrodynamic description of the interaction between a two-level atom and a nearly resonant electromagnetic field with a finite bandwidth spectrum. ${ }^{7}$ We include the phenomenological treatment of external laser phase fluctuations not because they play any essential role in our theory, but because we want to bring out explicitly that their effects on the relevant spectral distributions are clearly distinguishable and qualitatively different from the fundamental effects we are searching for.

According to conventional quantum mechanics, the isolated system composed of the atom and the field is always in a pure state and evolves according to the Schroedinger equation of motion. The Hamiltonian operator is written in the electric-dipole approximation neglecting the fieldfield coupling term. The driving field is assumed to be in a monochromatic coherent state with steady amplitude and a phase that performs a random walk with a diffusion time $1 / \lambda^{\prime}$ corresponding to an effective bandwidth $\lambda^{\prime}$ of the laser beam (Kimble and Mandel, 1977). Before averaging over the laser phases, the equations of motion for the reduced atomic state operator under the rotating-wave approximation (Ackerhalt and Eberly, 1974; Kimble and Mandel, 1975, 1977; Cohen-Tannoudji and Reynaud, 1977; Mollow, 1972; 1975; 1981), may be written as

$$
\begin{gathered}
\frac{d \alpha(t)}{d t}+\left[\beta+i\left(\omega_{0}-\gamma\right)\right] \alpha(t)+i \Omega \varepsilon(t) r_{3}(t) / 2=0 \\
\frac{d r_{3}(t)}{d t}+2 \beta\left[r_{3}(t)+1\right]+i \Omega\left[\varepsilon^{*}(t) \alpha(t)-\varepsilon(t) \alpha^{*}(t)\right]=0
\end{gathered}
$$

where $\Omega=2 \mu \cdot \mathbf{a} \omega_{0} / \hbar$ is the Rabi broadening parameter, $\hbar \omega_{0}$ is the energy difference between the two free-atom energy levels, $\mu$ is the atomic transition dipole moment matrix element between the two levels, $a$ is the real steady amplitude of the driving field, $\beta=2 \mu \cdot \mu \omega_{0}^{3} / 3 \hbar c^{3} 4 \pi \varepsilon_{0}$ is one-half of the Einstein $A$ coefficient (natural decay rate), $\gamma$ is the Lamb shift,

$$
\varepsilon(t)=e^{-\omega_{1} t+i \phi(t)}
$$

is the time dependence of the eigenvalue of the positive frequency part of the free-field vector potential corresponding to the monochromatic coherent state with frequency $\omega_{1}$ and phase $\phi(t)$, with random walk characteristic function (Kimble and Mandel, 1977)

$$
\left\langle\left\langle e^{i[\phi(t \pm \tau)-\phi(t)] x}\right\rangle=e^{-\lambda^{\prime} \tau x^{2}}, \quad \tau \geq 0\right.
$$

${ }^{7}$ We follow closely the treatments in Mollow $(1972,1975)$ and Kimble and Mandel (1977). 
where $\lambda^{\prime}$ is the effective bandwidth of the driving laser field with spectrum centered at frequency $\omega_{1}$. Equations (8) are rigorously valid in the limit as $\beta / \omega_{0},\left|\omega_{1}-\omega_{0}\right| / \omega_{0}$, and $\Omega / \omega_{0}$ approach zero (Mollow, 1972; 1975).

Comparison between the left-hand sides of equations (5) and (8) shows that the presence of the atomic dipole's own radiation effectively modifies (radiation reaction) the atom's (reduced) characteristics so as to produce a finite decay rate and a shift in the transition frequency (Ackerhalt et al., 1973).

\section{APPROXIMATE EQUATIONS FOR NONIDEMPOTENT ATOM-FIELD STATES}

We now investigate the consequences of our assumption that the composite isolated atom-field system may exist in nonmechanical states that must be described by nonidempotent state operators evolving according to the general nonlinear equation of motion of quantum thermodynamics. As a first model, in this paper we assume that the corresponding effect on the time evolution of the reduced atomic state operator is well approximated by adding to the right-hand side of equations (8) the right-hand side of equations (5), with $\varepsilon$ substituted by $\varepsilon(t)$ [equation (9)]. It is beyond our present objectives to justify rigorously the foregoing assumption in terms of our general nonlinear dynamics. We simply emphasize that our preliminary results based on this assumption are interesting and intriguing enough to stimulate further more rigorous investigations.

We will write our subsequent results in terms of the following set of dimensionless parameters:

$$
\begin{aligned}
\xi & =\beta / \omega_{0} \\
\eta & =\Omega / \sqrt{2} \beta \\
\delta & =1 / \beta \tau \\
\lambda & =\lambda^{\prime} / \beta \\
\mu & =\omega_{1} / \beta \\
\theta & =\left(\omega_{1}-\omega_{0}+\gamma\right) / \beta \\
\nu & =\left(\omega-\omega_{1}\right) / \beta \\
\Omega^{\prime} & =\left(2 \eta^{2}+\theta^{2}\right)^{1 / 2}
\end{aligned}
$$

with the assumption that $\beta, \Omega, \Omega / \tau \beta \ll \omega_{0}(\xi, \xi \eta, \delta \xi \eta \ll 1){ }^{8}$

\footnotetext{
${ }^{8}$ Under these approximations, the matrix elements $[\mathbf{A}]_{13}=[\mathbf{A}]_{31}=-\Delta \xi^{2} \eta^{2}$ [equation (18)] can for all purposes be set equal to zero.
} 
The governing equations become

$$
\begin{gathered}
\frac{1}{\beta} \frac{d \alpha(t)}{d t}+(1+i \mu-i \theta) \alpha(t)+i \eta \varepsilon(t) \frac{r_{3}(t)}{\sqrt{2}} \\
=-\Delta \alpha(t)-\Delta \xi \eta \varepsilon(t) \frac{r_{3}(t)}{\sqrt{2}}+\Delta \xi^{2} \eta^{2} \varepsilon^{2}(t) \alpha^{*}(t) \\
\frac{1}{\sqrt{2} \beta} \frac{d r_{3}(t)}{d t}+i \eta \varepsilon^{*}(t) \alpha(t)+2 \frac{r_{3}(t)}{\sqrt{2}}+\sqrt{2}-i \eta \varepsilon(t) \alpha^{*}(t) \\
=-\Delta \xi \eta \varepsilon^{*}(t) \alpha(t)-\Delta \xi \eta \varepsilon(t) \alpha^{*}(t)
\end{gathered}
$$

where we defined the variable

$$
\Delta=\delta K\left(\alpha, r_{3}\right)
$$

with $K\left(\alpha, r_{3}\right)$ given by equations (6) and (7).

Equations (12) reduce to the quantum electrodynamic case for $\delta=0$. They are nonlinear for $\delta>0$. Whether $\delta=0$ or $\delta>0$, as long as the exciting field amplitude does not vanish $(\eta>0)$, equations (12) admit of a steady state nonequilibrium solution in the long-time limit $t \rightarrow \infty$.

From here on, we will study only the long-time limit, asymptotic solutions of equations (12). In that limit the function $\Delta$ becomes a constant and the equations become linear. Thus, we will first determine the steady state expressions for $\alpha$ and $r_{3}$ as functions of $\Delta$ [equation (41)] and then use equation (12c) to solve for $\Delta, \alpha$, and $r_{3}$.

In the long-time limit, because the dynamic equations become linear, the substitutions

$$
\alpha(t) \leftrightarrow b(t), \quad r_{3}(t) / \sqrt{2} \leftrightarrow \sqrt{2} R_{3}(t), \quad \alpha^{*}(t) \leftrightarrow b^{\dagger}(t)
$$

allow us to pass to the Heisenberg picture which will result particularly useful to evaluate correlation functions.

We now introduce useful shorthand notation:

$$
\begin{gathered}
\mathbf{X}(t)=\left|\begin{array}{c}
b(t) \\
\sqrt{2} R_{3}(t) \\
b^{\dagger}(t)
\end{array}\right|, \quad \mathbf{U}=\left|\begin{array}{l}
0 \\
I \\
0
\end{array}\right| \\
\mathbf{x}(t)=\langle\mathbf{X}(t)\rangle=\left|\begin{array}{c}
\alpha(t) \\
r_{3}(t) / \sqrt{2} \\
\alpha^{*}(t)
\end{array}\right|, \quad \mathbf{u}=\left|\begin{array}{l}
0 \\
1 \\
0
\end{array}\right| \\
\mathbf{g}_{e}(t, \tau)=\left\langle b^{\dagger}(t) \mathbf{X}(t+\tau)\right\rangle \\
\mathbf{g}_{a}(t, \tau)=\left\langle\left[\mathbf{X}(t+\tau), b^{\dagger}(t)\right]\right\rangle
\end{gathered}
$$




$$
\begin{gathered}
\mathbf{A}=\left|\begin{array}{ccc}
1+\Delta-i \theta & i \eta(1-i \Delta \xi) & -\Delta \xi^{2} \eta^{2} \\
i \eta(1-i \Delta \xi) & 2 & -i \eta(1+i \Delta \xi) \\
-\Delta \xi^{2} \eta^{2} & -i \eta(1+i \Delta \xi) & 1+\Delta+i \theta
\end{array}\right| \\
\mathbf{E}(t)=\left|\begin{array}{ccc}
\varepsilon(t) & 0 & 0 \\
0 & 1 & 0 \\
0 & 0 & \varepsilon^{*}(t)
\end{array}\right| \\
\mathbf{F}(t, \tau)=\varepsilon(t) e^{i[\phi(t+\tau)-\phi(t)]} E^{*}(t+\tau) \\
\mathbf{B}=\left|\begin{array}{ccc}
i \mu & 0 & 0 \\
0 & 0 & 0 \\
0 & 0 & -i \mu
\end{array}\right|
\end{gathered}
$$

Equations (12a) and (12b) may be rewritten as

$$
\frac{1}{\beta} \dot{x}(t)=-\mathrm{E}(t) \cdot[\mathrm{A}+\mathrm{B}] \cdot \mathrm{E}^{*}(t) \cdot \mathbf{x}(t)-\sqrt{2} \mathbf{u}
$$

and, in the long-time limit Heisenberg picture, as

$$
\frac{1}{\beta} \dot{\mathbf{X}}(t)=-\mathrm{E}(t) \cdot[\mathrm{A}+\mathrm{B}] \cdot \mathrm{E}^{*}(t) \cdot \mathbf{X}(t)-\sqrt{2} \mathrm{U}
$$

Moreover, using equation (23), we find

$$
\begin{gathered}
\frac{1}{\beta} \frac{\partial}{\partial \tau} \mathbf{g}_{e}(t, \tau)=-\mathrm{E}(t+\tau) \cdot[\mathrm{A}+\mathrm{B}] \cdot \mathrm{E}^{*}(t+\tau) \cdot \mathrm{g}_{e}(t, \tau)-\sqrt{2} \alpha^{*}(t) \mathbf{u} \\
\frac{1}{\beta} \frac{\partial}{\partial \tau} \mathbf{g}_{a}(t, \tau)=-\mathrm{E}(t+\tau) \cdot[\mathrm{A}+\mathrm{B}] \cdot \mathrm{E}^{*}(t+\tau) \cdot \mathbf{g}_{a}(t, \tau)
\end{gathered}
$$

Next, we introduce the slowly varying functions

$$
\begin{aligned}
\mathbf{x}_{s}(t) & =\mathrm{E}^{*}(t) \cdot \mathbf{x}(t) \\
\mathbf{g}_{e s}(t, \tau) & =\mathbf{F}(t, \tau) \cdot \mathbf{g}_{e}(t, \tau) \\
\mathbf{g}_{a s}(t, \tau) & =\mathrm{F}(t, \tau) \cdot \mathbf{g}_{a}(t, \tau)
\end{aligned}
$$

where equations (20) and (27) have been selected so that $[F(t, \tau)]_{11}=e^{i \omega_{1} \tau}$ is phase independent and, therefore, the interesting two-time atomic correlation functions (cf. Sections 6 and 7) are given by

$$
\begin{aligned}
& \left\langle\left[\mathbf{g}_{e}(t, \tau)\right]_{1}\right\rangle=e^{-i \omega_{1} \tau}\left\langle\left\langle\left[\mathbf{g}_{e s}(t, \tau)\right]_{1}\right\rangle\right\rangle \\
& \left\langle\left\langle\left[\mathbf{g}_{a}(t, \tau)\right]_{1}\right\rangle\right\rangle=e^{-i \omega_{1} \tau}\left\langle\left[\left[\mathbf{g}_{a s}(t, \tau)\right]_{1}\right\rangle\right\rangle
\end{aligned}
$$


Equations (22), (24), and (25) become

$$
\begin{aligned}
\frac{1}{\beta} \dot{\mathbf{x}}_{s}(t)= & -\left[\mathbf{A}+\mathbf{B}-\frac{1}{\beta} \mathbf{E}(t) \cdot \dot{E}^{*}(t)\right] \cdot \mathbf{x}_{s}(t)-\sqrt{2} \mathbf{u} \\
\frac{1}{\beta} \frac{\partial}{\partial \tau} \mathbf{g}_{e s}(t, \tau)= & -\left[\mathbf{A}+\mathbf{B}-\frac{1}{\beta} \mathrm{F}^{*}(t+\tau) \cdot \frac{\partial}{\partial \tau} \mathrm{F}(t+\tau)\right] \cdot \mathbf{g}_{e s}(t, \tau) \\
& -\sqrt{2} \alpha_{s}^{*}(t) e^{i[\phi(t+\tau)-\phi(t)]} \mathbf{u} \\
\frac{1}{\beta} \frac{\partial}{\partial \tau} \mathbf{g}_{a s}(t, \tau)= & -\left[\mathbf{A}+\mathbf{B}-\frac{1}{\beta} \mathrm{F}^{*}(t+\tau) \cdot \frac{\partial}{\partial \tau} \mathrm{F}(t+\tau)\right] \cdot \mathbf{g}_{a s}(t, \tau)
\end{aligned}
$$

where $\alpha_{s}^{*}(t)=\varepsilon(t) \alpha^{*}(t)$.

We now take the average $\langle\langle\cdot\rangle$ over the ensemble of phases, assuming independence of the slowly varying atomic variables at time $t$ from phase changes at subsequent times $t+\tau(\tau>0)$ (Kimble and Mandel, 1977) and, hence, also from the phase rates of change $\dot{\phi}(t)$ and $\dot{\phi}(t+\tau)$. We also use the relation

$$
\left\langle\langle i x \dot{\phi}(t)\rangle=\frac{\partial}{\partial \tau}\left\langle\left.\left\langle e^{i[\phi(t+\tau)-\phi(t)] x}\right\rangle\right|_{\tau=0}=-\lambda \beta x^{2}\right.\right.
$$

obtained from equation (10).

Equations (30), (31), and (32) become

$$
\begin{gathered}
\frac{1}{\beta} \dot{\boldsymbol{x}}_{s}(t)=-[\mathbf{A}+\mathbf{C}] \cdot \boldsymbol{X}_{s}(t)-\sqrt{2} \mathbf{u} \\
\frac{1}{\beta} \frac{\partial}{\partial \tau} \Gamma_{e s}(t, \tau)=-[\mathbf{A}+\mathbf{D}] \cdot \Gamma_{e s}(t, \tau)-\sqrt{2} \alpha_{s}^{*}(t) e^{-\lambda \beta \tau} \mathbf{u} \\
\frac{1}{\beta} \frac{\partial}{\partial \tau} \Gamma_{a s}(t, \tau)=-[\mathbf{A}+\mathrm{D}] \cdot \Gamma_{a s}(t, \tau)
\end{gathered}
$$

where

$$
\begin{gathered}
\boldsymbol{\chi}_{s}(t)=\left\langle\left\langle\mathbf{x}_{s}(t)\right\rangle\right\rangle \\
\Gamma_{e s}(t, \tau)=\left\langle\left\langle\mathbf{g}_{e s}(t, \tau)\right\rangle\right\rangle \\
\Gamma_{e s}(t, \tau)=\left\langle\left\langle\mathbf{g}_{a s}(t, \tau)\right\rangle\right\rangle \\
\mathbf{C}=\left|\begin{array}{lll}
\lambda & 0 & 0 \\
0 & 0 & 0 \\
0 & 0 & \lambda
\end{array}\right|, \quad \mathrm{D}=\left|\begin{array}{ccc}
0 & 0 & 0 \\
0 & \lambda & 0 \\
0 & 0 & 4 \lambda
\end{array}\right|
\end{gathered}
$$


For any value of $\Delta$, the eigenvalues of matrices $(A+C)$ and $(A+D)$ have positive real part. Hence, the long-time limit, steady state solutions of equations (34), (35), and (36) are

$$
\begin{gathered}
\boldsymbol{\chi}_{s}(\infty)=-\sqrt{2}\left[(\mathbf{A}+\mathbf{C})^{-1}\right] \cdot \mathbf{u} \\
\boldsymbol{\Gamma}_{e s}(\infty, \tau)=e^{-\beta \lambda \tau} \mathbf{G}(\infty)+\left[e^{-\beta(\mathbf{A}+\mathrm{D}) \tau}\right] \cdot\left[\boldsymbol{\Gamma}_{e s}(\infty, 0)-\mathbf{G}(\infty)\right] \\
\boldsymbol{\Gamma}_{a s}(\infty, \tau)=\left[e^{-\beta(\mathbf{A}+\mathbf{D}) \tau}\right] \cdot \boldsymbol{\Gamma}_{a s}(\infty, 0)
\end{gathered}
$$

where

$$
\begin{array}{r}
\mathbf{G}(\infty)=-\sqrt{2}\left\langle\left\langle\alpha_{s}^{*}(\infty)\right\rangle\right\rangle\left[(\mathbf{A}+\mathbf{D}-\lambda \mathbf{I})^{-1}\right] \cdot \mathbf{u} \\
\boldsymbol{\Gamma}_{e s}(\infty, 0)=\left|\begin{array}{c}
\left(\left\langle r_{3}(\infty)\right\rangle+1\right) / 2 \\
-\left\langle\left\langle\alpha_{s}^{*}(\infty)\right\rangle / \sqrt{2}\right. \\
0
\end{array}\right| \\
\boldsymbol{\Gamma}_{a s}(\infty, 0)=\left|\begin{array}{c}
-\left\langle\left\langle\boldsymbol{r}_{3}(\infty)\right\rangle\right\rangle \\
\sqrt{2}\left\langle\left\langle\alpha_{s}^{*}(\infty)\right\rangle\right. \\
0
\end{array}\right|
\end{array}
$$

and we used the fact that ${ }^{4}$

$$
\begin{gathered}
\mathbf{g}_{e}(\infty, 0)=\left\langle b^{\dagger}(\infty) \mathbf{X}(\infty)\right\rangle=\left\langle\left|\begin{array}{c}
R_{3}(\infty)+I / 2 \\
-b^{\dagger}(\infty) / \sqrt{2} \\
0
\end{array}\right|\right\rangle=\left|\begin{array}{c}
\left(r_{3}(\infty)+1\right) / 2 \\
-\alpha^{*}(\infty) / \sqrt{2} \\
0
\end{array}\right| \\
\mathbf{g}_{a}(\infty, 0)=\left\langle\left[\mathbf{X}(\infty), b^{\dagger}(\infty)\right]\right\rangle=\left\langle\left|\begin{array}{c}
-2 R_{3}(\infty) \\
\sqrt{2} b^{\dagger}(\infty) \\
0
\end{array}\right|\right\rangle=\left|\begin{array}{c}
-r_{3}(\infty) \\
\sqrt{2} \alpha^{*}(\infty) \\
0
\end{array}\right|
\end{gathered}
$$

The steady state solution [equation (41)] is a function of the variable $\Delta$ (equation 12c) which in the approximations adopted (rotating wave, $\Omega \ll \omega_{0}$ ) may be rewritten as

$$
\Delta=\delta \frac{1-\langle\langle r(\infty)\rangle\rangle^{2}}{1-\left\langle\left\langle r_{3}(\infty)\right)\right\rangle^{2}} \frac{1}{2\langle\langle r(\infty)\rangle\rangle} \ln \frac{1+\langle\langle r(\infty)\rangle\rangle}{1-\langle\langle r(\infty)\rangle\rangle}
$$

with

$$
\begin{aligned}
& \left\langle r_{3}(\infty)\right\rangle=-\frac{(1+\Delta+\lambda)^{2}+\theta^{2}}{(1+\Delta+\lambda)^{2}+\theta^{2}+\eta^{2}(1+\Delta+\lambda+2 \Delta \xi \theta)} \\
& \langle r(\infty)\rangle\rangle^{2}=\frac{\left[(1+\Delta+\lambda)^{2}+\theta^{2}\right]\left[(1+\Delta+\lambda)^{2}+\theta^{2}+2 \eta^{2}\right]}{\left[(1+\Delta+\lambda)^{2}+\theta^{2}+\eta^{2}(1+\Delta+\lambda+2 \Delta \xi \theta)\right]^{2}}
\end{aligned}
$$

For each given value of $\xi, \eta, \theta$, and $\lambda$, equations (49)-(51) can be solved for $\Delta$ as a function of $\delta$. As $\eta$ varies from $10^{-4}$ to $10^{2}$, Figure 1 shows the 

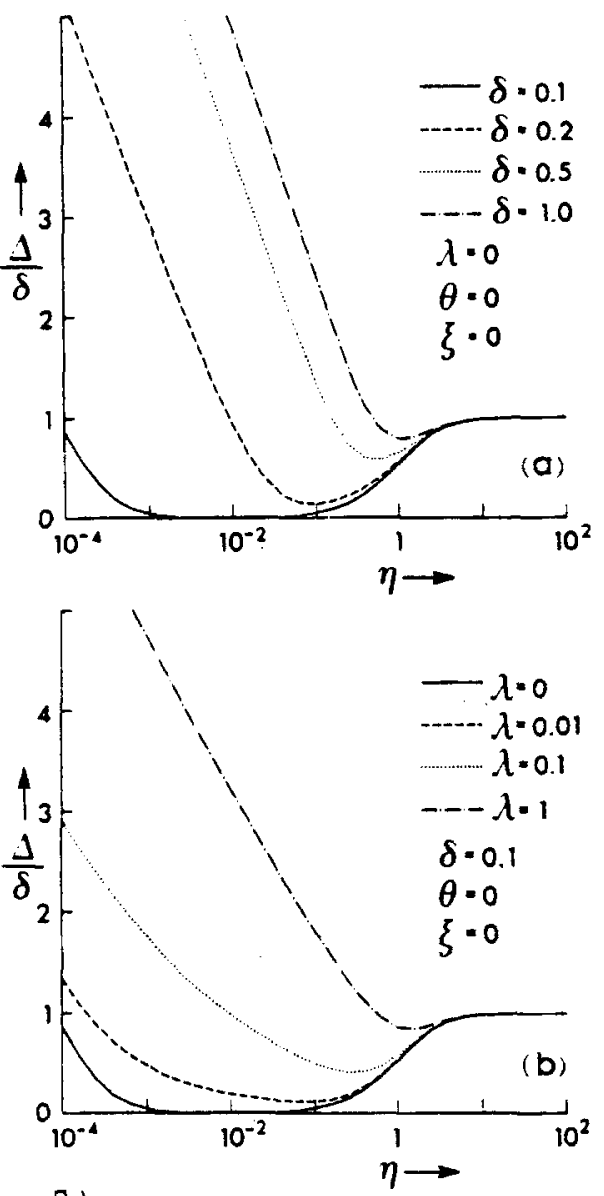

Fig. 1. Steady state values of $\Delta / \delta=$ $K\left(\alpha, r_{3}\right)$ as a function of driving field strength $\eta=\Omega / \sqrt{2} \beta$. (a) Dependence on the internal redistribution parameter $\delta=$ $1 / \beta \tau$. (b) Dependence on the driving field effective bandwidth $\lambda=\lambda^{\prime} / \beta$. (c) Dependence on the detuning parameter $\theta=$ $\left(\omega_{1}-\omega_{0}+\gamma\right) / \beta$.

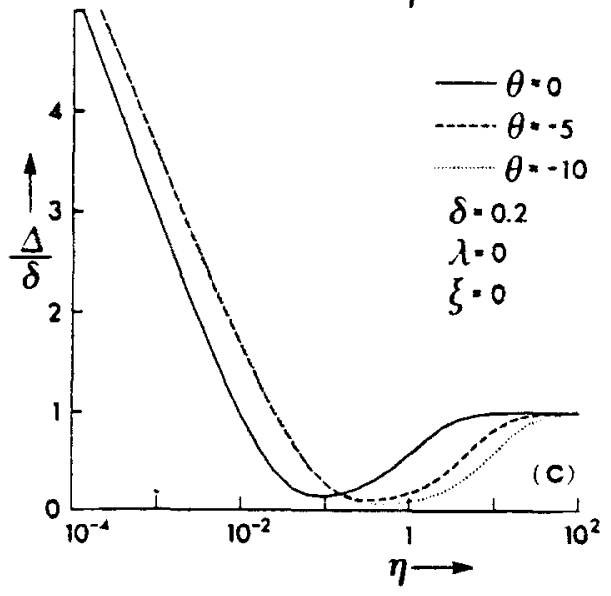


dependences of $\Delta$ on $\delta$ (part a), on $\lambda$ (part b), and on $\theta$ (part c). In the weak-field limit $(\eta \rightarrow 0)$, the ratio $\Delta / \delta$ tends to $+\infty$, i.e., the steady state effect of internal atomic relaxation is amplified. However, the emission intensity in that limit equals zero, as the steady state solution approaches the idempotent equilibrium state with $r_{3}=-1$. In the strong-field limit $(\eta \rightarrow \infty)$, instead, $\Delta$ tends to $\delta$ as the steady state solution approaches the nonidempotent state $\mathbf{r}=0$.

The steady state fluorescent light intensity is proportional to

$$
\left.\frac{1}{2}\left(\left\langle r_{3}(\infty)\right\rangle\right)+1\right)=\frac{1}{2} \frac{\eta^{2}(1+\lambda+\Delta+2 \Delta \xi \theta)}{(1+\lambda+\Delta)^{2}+\theta^{2}+\eta^{2}(1+\lambda+\Delta+2 \Delta \xi \theta)}
$$

This expression can be interpreted as the absorption spectrum. The effect of our atomic redistribution mechanism on the absorption spectrum is shown in Figure 2 for a weak-field case (part a) and a strong-field case (part b). The steady state fluorescent light intensity is evaluated from equation (52) with $\Delta$ obtained from equations (49), (50), and (51) for each value of $\theta$. To display both the broadening introduced by a nonzero value of parameter $\delta$, and the imperceptible asymmetry introduced by a nonzero value of paramenter $\xi$ (which for optical transitions is of order $10^{-6}$ ), we have set $\xi$ to an unrealistic value 0.01 and plotted the spectra by setting the maximum value equal to 1.0. The actual maxima for $\eta=0.1$ (Figure 2a) are 0.3167 , 0.1666 , and 0.0815 , and for $\eta=10$ (Figure $2 b$ ) are $0.03167,0.03256,0.03617$, corresponding to $\delta$ equal to $0.0,0.5$, and 1.0 , respectively. Slight asymmetries in the absorption spectrum have been observed experimentally, (Schuda et al., 1974; Wu et al., 1975; Hartig et al., 1976; Grove et al., 1977; Wu et al., 1977; Citron et al., 1977; Ezekiel and Wu, 1977; Cresser et al., 1982; Peuse et al., 1982) but it seems unlikely that the asymmetry predicted here could be actually detectable. What could be disclosed by a quantitative highresolution experimental study is the broadening effect due to our internal redistribution parameter $\delta$ or, at least, bounds to its value could be determined. As the laser bandwidth parameter $\lambda$ contributes to broadening the absorption spectrum exactly like parameter $\delta$, it is important that $\lambda$ be predetermined independently for the given laser beam.

\section{RESONANCE FLUORESCENCE}

The time-dependent spectral distribution of the fluorescence (photon emission) at points in the far field not exposed to the driving field and after the propagation time delay, is proportional to the Fourier integral (Ackerhalt and Eberly, 1974; Kimble and Mandel, 1975; 1977; Cohen-Tannoudji and 


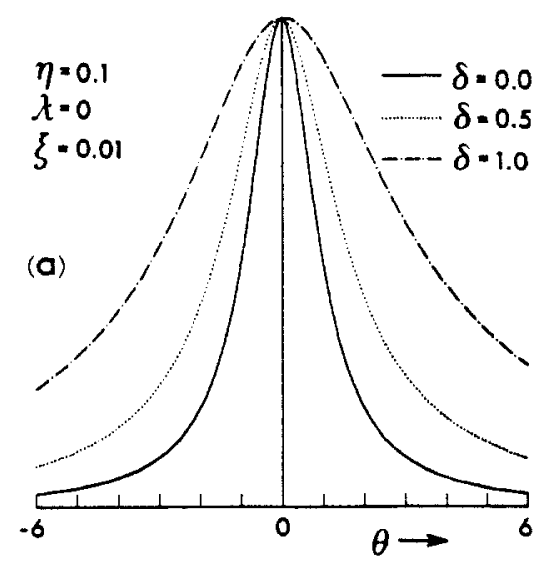

Fig. 2. Absorption spectrum $\left(\left\langle r_{3}(\infty)\right\rangle+1\right) / 2$ as a function of the detuning parameter $\theta$ for monochromatic excitation $(\lambda=0)$. (a) Weak driving field $(\eta=0.1)$. (b) Strong driving field $(\eta=10)$. To amplify the imperceptible asymmetry, the spectra are not normalized and the value of $\xi$ is unrealistically high.

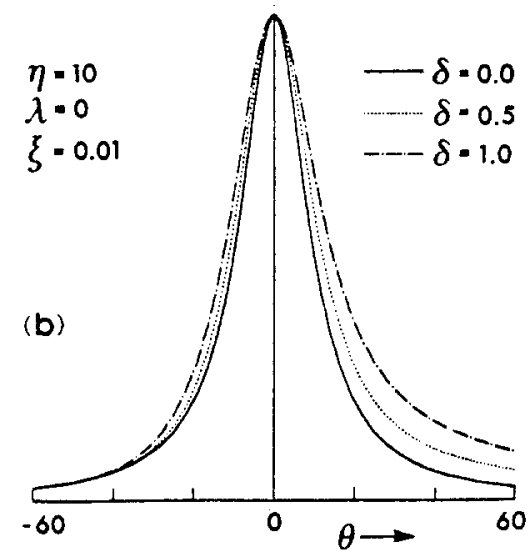

Reynaud, 1977; Mollow, 1972; 1975; 1981)

$$
\int_{0}^{t} d \tau\left\langle\left\langle\left[\mathbf{g}_{e}(t-\tau, \tau)\right]_{1}\right\rangle e^{i \omega \tau}+\right.\text { c.c. }
$$

which in the steady state long-time limit becomes

$$
\Phi_{e}(\nu)=\int_{0}^{\infty} d \tau\left[\Gamma_{e s}(\infty, \tau)\right]_{1} e^{i \beta \nu \tau}+\text { c.c. }
$$

where $\nu=\left(\omega-\omega_{1}\right) / \beta$ and we used equations (28), (38), and the fact that at steady state $\Gamma_{e s}(t, \tau)$ becomes independent of the first argument $t$. With 
the help of equations (41), (42), (44), and (45), we find

$$
\begin{gathered}
\Gamma_{e s}(\infty, 0)=\left|\begin{array}{c}
\left(1-2\left[(\mathbf{A}+\mathbf{C})^{-1}\right]_{22}\right) / 2 \\
{\left[(\mathbf{A}+\mathbf{C})^{-1}\right]_{32}} \\
0
\end{array}\right| \\
\mathbf{G}(\infty)=2\left[(\mathbf{A}+\mathbf{C})^{-1}\right]_{32}\left|\begin{array}{c}
{\left[(\mathbf{A}+\mathbf{D}-\lambda \mathbf{I})^{-1}\right]_{12}} \\
{\left[\left(\mathbf{A}+\mathbf{D}-\lambda \mathbf{I}^{-1}\right]_{22}\right.} \\
{\left[\left(\mathbf{A}+\mathbf{D}-\lambda \mathbf{I}^{-1}\right)\right]_{32}}
\end{array}\right| \\
\beta \int_{0}^{\infty} d \tau e^{i \beta \nu \tau} \boldsymbol{\Gamma}_{e s}(\infty, \tau)=\frac{1}{\lambda-i \nu} \mathbf{G}(\infty) \\
+\left[(\mathbf{A}+\mathbf{D}-i \nu \mathbf{l})^{-1}\right] \cdot\left[\boldsymbol{\Gamma}_{e s}(\infty, 0)-\mathbf{G}(\infty)\right]
\end{gathered}
$$

After lengthy, but sraightforward manipulations, we find the following four equivalent expressions for the spectral density of the fluorescent light

$$
\begin{aligned}
\beta \Phi_{3}(\nu) & =\frac{c}{2}\left\{\frac{1}{\lambda-i \nu}-\frac{n(-i \nu)}{(\lambda-i \nu) f(-i \nu)}+\text { c.c. }\right\} \\
& =\frac{c}{2}\left\{\frac{d_{0}}{\lambda-i \nu}+\sum_{k=1}^{3} \frac{d_{k}}{p_{k}+i \nu}+\text { c.c. }\right\} \\
& =c\left\{\frac{\lambda \operatorname{Re} d_{0}-\nu \operatorname{Im} d_{0}}{\lambda^{2}+\nu^{2}}+\sum_{k=1}^{3} \frac{\operatorname{Re} p_{k} \operatorname{Re} d_{k}+\left(\nu+\operatorname{Im} p_{k}\right) \operatorname{Im} d_{k}}{\left(\operatorname{Re} p_{k}\right)^{2}+\left(\nu+\operatorname{Im} p_{k}\right)^{2}}\right\} \\
& =c\left\{\frac{\lambda}{\lambda^{2}+\nu^{2}}-\frac{\lambda[u(\nu) a(\nu)+v(\nu) b(\nu)]+\nu[v(\nu) a(\nu)-u(\nu) b(\nu)]}{\left(\lambda^{2}+\nu^{2}\right)\left[u(\nu)^{2}+v(\nu)^{2}\right]}\right\}
\end{aligned}
$$

where

$$
\begin{aligned}
c=\frac{\eta^{2}}{(1+\Delta+\lambda)^{2}+\theta^{2}+\eta^{2}(1+\Delta+\lambda+2 \Delta \xi \theta)} \\
f(p)=\operatorname{det}(\mathbf{A}+\mathrm{D}+p \mathbf{I})=\left(p-p_{1}\right)\left(p-p_{2}\right)\left(p-p_{3}\right) \\
=2 \eta^{2}[1+2 \lambda+p+\Delta(1+2 \xi \theta-4 i \xi \lambda)] \\
+(2+\lambda+p)(1+\Delta+4 \lambda+p+i \theta)(1+\Delta+p-i \theta) \\
=p^{3}+(4+2 \Delta+5 \lambda) p^{2}+\left[2 \eta^{2}+(1+\Delta+2 \lambda)(5+\Delta+2 \lambda)\right. \\
\left.+2 \lambda(1+\Delta)+\theta^{2}-4 i \lambda \theta\right] p \\
+2 \eta^{2}[1+2 \lambda+\Delta(1+2 \xi \theta-4 i \xi \lambda)] \\
+(2+\lambda)\left[(1+\Delta)(1+\Delta+4 \lambda)+\theta^{2}-4 i \lambda \theta\right]
\end{aligned}
$$


$p_{1}, p_{2}, p_{3}$ are the roots of the cubic equation $f(p)=0$,

$$
\begin{aligned}
n(p)= & \eta^{2}\{2+p-\lambda(p-3+\lambda)-\Delta p[1+2 \xi \theta+2 i \xi(1+\Delta+\lambda)] \\
& -\Delta \lambda[1+2 i \xi(5+\Delta+\lambda)]\} \\
& -(2+\lambda+p)(1+\Delta+4 \lambda+p+i \theta)[\lambda(2+\lambda+p)+\Delta(\lambda+p)(1+2 \xi \theta)]
\end{aligned}
$$

$$
\begin{aligned}
= & -[\lambda+\Delta(1+2 \xi \theta)] p^{3}-[\lambda(5+\Delta+6 \lambda+i \theta)+\Delta(1+2 \xi \theta) \\
& \cdot(3+\Delta+6 \lambda+i \theta)] p^{2} \\
& -\left\{2 i \lambda \theta(2+\lambda)+\Delta(1+2 \xi \theta)\left[2+12 \lambda+9 \lambda^{2}+2(\Delta+i \theta)(1+\lambda)\right]\right. \\
& \left.-\eta^{2}[1-\lambda-\Delta(1+2 \xi \theta+2 i \xi(1+\Delta+\lambda))]\right\} p \\
& -\lambda(2+\lambda)(1+\Delta+4 \lambda+i \theta)[2+\lambda+\Delta(1+2 \xi \theta)] \\
& +\eta^{2}[2(1+\Delta)+\lambda(3-\Delta-\lambda)-2 i \Delta \xi \lambda(5+\Delta+\lambda)] \\
d_{0}= & \frac{f(-\lambda)-n(-\lambda)}{f(-\lambda)} \\
= & \frac{(1+\Delta+3 \lambda+i \theta)(1+\Delta+\lambda-i \theta)+\eta^{2} \Delta \xi \theta(2+\lambda)}{(1+\Delta+3 \lambda+i \theta)(1+\Delta-\lambda-i \theta)+\eta^{2}[1+\lambda+\Delta(1+2 \xi \theta-4 i \xi \lambda)]}
\end{aligned}
$$$$
d_{k}=\frac{n\left(p_{k}\right)}{\left(\lambda+p_{k}\right) f^{\prime}\left(p_{k}\right)}=\frac{n\left(p_{k}\right)}{\left(\lambda+p_{k}\right)\left(p_{k}-p_{j}\right)\left(p_{k}-p_{h}\right)} \quad j \neq k, h \neq k
$$

$$
f^{\prime}(p)=\frac{d f(p)}{d p}
$$

$$
\begin{aligned}
u(\nu)= & \operatorname{Re}[f(-i \nu)]=-(4+2 \Delta+5 \lambda) \nu^{2}-4 \lambda \theta \nu+2 \eta^{2}[1+2 \lambda+\Delta(1+2 \xi \theta)] \\
& +(2+\lambda)\left[(1+\Delta)(1+\Delta+4 \lambda)+\theta^{2}\right]
\end{aligned}
$$

$$
v(\nu)=\operatorname{Im}[f(-i \nu)]=\nu^{3}-\left[(1+\Delta+2 \lambda)(5+\Delta+2 \lambda)+2 \lambda(1+\Delta)+\theta^{2}+2 \eta^{2}\right] \nu
$$

$$
-4 \lambda\left[(2+\lambda) \theta+2 \eta^{2} \Delta \xi\right]
$$

$$
\begin{aligned}
a(\nu)= & \operatorname{Re}[n(-i \nu)]=[\lambda(5+\Delta+6 \lambda)+\Delta(1+2 \xi \theta)(3+\Delta+6 \lambda)] \nu^{2} \\
& -2\left\{\theta[\lambda(2+\lambda)+\Delta(1+2 \xi \theta)(1+\lambda)]+\eta^{2} \Delta \xi(1+\Delta+\lambda)\right\} \nu \\
& -\lambda(2+\lambda)(1+\Delta+4 \lambda)[2+\lambda+\Delta(1+2 \xi \theta)] \\
& +\eta^{2}\left[2+3 \lambda-\lambda^{2}+\Delta(2-\lambda)\right]
\end{aligned}
$$

$$
\begin{aligned}
b(\nu)= & \operatorname{Im}[n(-i \nu)]=[\lambda+\Delta(1+2 \xi \theta)](\theta-\nu) \nu^{2}+\{\lambda(2+\lambda)(4+2 \Delta+9 \lambda) \\
& \left.+\Delta(1+2 \xi \theta)\left[2+12 \lambda+9 \lambda^{2}+2 \Delta(1+\lambda)\right]-\eta^{2}[1-\lambda-\Delta(1+2 \xi \theta)]\right\} \nu \\
& -\lambda \theta(2+\lambda)[2+\lambda+\Delta(1+2 \xi \theta)]-2 \eta^{2} \Delta \xi \lambda(5+\Delta+\lambda)
\end{aligned}
$$


The spectral density is a superposition of four pseudo-Lorentzian terms, each of the form

$$
\frac{x_{k} r_{k}+y_{k}\left(\nu-\nu_{k}\right)}{r_{k}^{2}+\left(\nu-\nu_{k}\right)^{2}}
$$

which, if $y_{k} \ll x_{k}$, has a peak centered approximately at

$$
\nu_{\max } \approx \nu_{k}+\frac{1}{2} \frac{y_{k}}{x_{k}} r_{k}
$$

with height approximately equal to $x_{k} / r_{k}$. The four terms of $\Phi_{e}(\nu)$ are centered at $\nu_{0}=0, \nu_{1}=-\operatorname{Im} p_{1}, \nu_{2}=-\operatorname{Im} p_{2}$, and $\nu_{3}=-\operatorname{Im} p_{3}$.

In the strong-field limit $\eta \gg 1, \lambda(\Delta \approx \delta$, Figure 1$)$ with not too strong detuning $(|\theta| \leq \eta)$, the roots of the cubic equation $f(p)=0$ are approximately given by

$$
\begin{aligned}
p_{1} & \approx \frac{2 \eta^{2}(1+\delta+2 \lambda)+\theta^{2}(2+\lambda)}{2 \eta^{2}+\theta^{2}}+4 i \lambda \frac{4 \eta^{4} \delta \xi-2 \eta^{2} \theta(2 \lambda+\delta)-\theta^{3}(2+\lambda)+\theta^{4}}{\left(2 \eta^{2}+\theta^{2}\right)^{2}} \\
p_{2,3} & \approx \frac{\eta^{2}[ \pm 2 \lambda \theta-(3+\delta+3 \lambda)]+\theta^{2}[ \pm \lambda \theta-(1+\delta+2 \lambda)]}{2 \eta^{2}+\theta^{2}} \pm i \Omega^{\prime}
\end{aligned}
$$

The first term of $\Phi_{e}(\nu)$ is centered at $\nu_{0}=0$ and represents elastically scattered light. The second term, centered at $\nu_{1}=-\operatorname{Im} p_{1}$, is very slightly shifted from 0 if the excitation bandwidth $\lambda \neq 0$. However, this shift is negligible with respect to the Stark shift of the third and fourth peaks centered at $\nu_{2,3}= \pm \Omega^{\prime}$. The spectral density at 0 and $\pm \Omega^{\prime}$ is approximately given by

$$
\begin{gathered}
\beta \Phi_{1}(0)=c \frac{\eta^{2}(1+\delta+\lambda)+\theta^{2}(2+\lambda) / \lambda}{2 \eta^{2}(1+\delta+2 \lambda)+\theta^{2}(2+\lambda)} \\
\beta \Phi_{1}\left( \pm \Omega^{\prime}\right)=c \frac{\eta^{2}(1+\delta+\lambda)-\left( \pm \Omega^{\prime} \theta-\theta^{2}\right)(\delta+\lambda)}{2 \eta^{2}(3+\delta+3 \lambda)+2 \theta^{2}(1+\delta+2 \lambda) \pm 4 \lambda \Omega^{\prime} \theta}
\end{gathered}
$$

On resonance $(\theta=0)$, the strong-field limit spectral density is symmetric, centered at the driving frequency $(\nu=0)$ and such that the ratio of the height of the central peak (excluding the elastic scattering contribution) to the height of the side peaks is approximately equal to

$$
(3+\delta+3 \lambda) /(1+\delta+2 \lambda)
$$

The effect of the irreversible internal relaxation parameter $\delta=1 / \beta \tau$ is shown in Figure 3a, where the central peak is truncated to magnify the effect of $\delta$ on the side peaks. The steady state values of $\Delta$ in Figure 3a are 0.0987, 


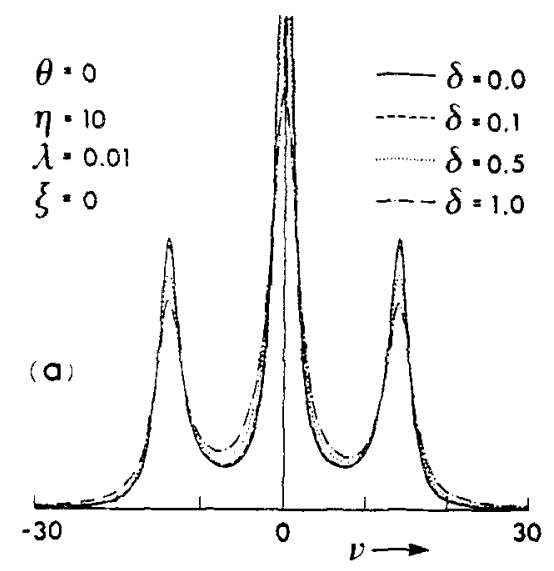

Fig. 3. Resonance fluorescence spectrum $\Phi_{e}(\nu)$ as a function of the dimensionless emission frequency $\nu=\left(\omega-\omega_{1}\right) / \beta$. Dependence on the internal redistribution parameter $\delta$ in the strong-field limit $(\eta=10)$ with nearly monochromatic excitation $(\lambda=0.01)$. (a) On resonance $(\theta=0)$. (b) Off resonance $(\theta=$ $-10)$.

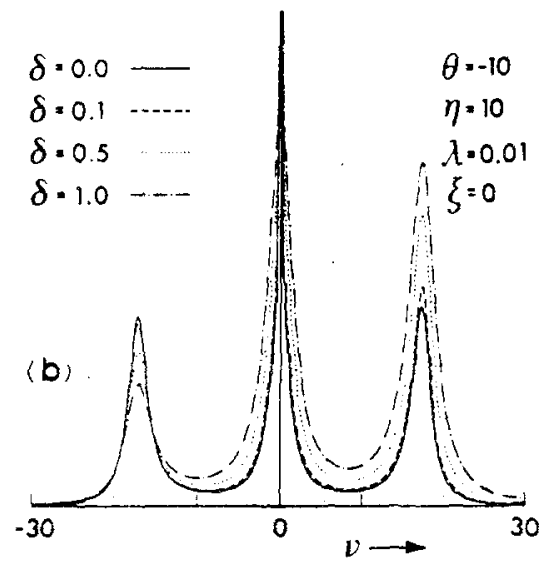

0.4935 , and 0.9873 for $\delta$ equal to $0.1,0.5$, and 1.0 , respectively. For $\delta=1.0$, the ratio of the height of the inelastic part of the central peak and the height of the side peaks is approximately $2: 1$, instead of the quantum electrodynamic $3: 1$. If the internal relaxation time constant $\tau$ is taken to be a universal constant, then our effect is negligible for atomic transitions with large values of $\beta$, whereas it is amplified for transitions with small values of $\beta$ (as long as $\left.\Omega / \tau \beta \ll \omega_{0}\right)$.

Off resonance $(\theta \neq 0)$, the strong-field limit spectral density is symmetric for $\lambda=0$ and $\delta=0$. For $\lambda \neq 0$, it becomes asymmetric (Kimble and Mandel, $1977)$ and, as $|\theta|$ is increased, one of the side peaks rapidly disappears and the other, centered at the atomic resonance frequency $\omega_{0}$, becomes dominant. For $\theta=-10$ and $\lambda=0.01$ (i.e., for almost monochromatic excitation), the curve in Figure $3 \mathrm{~b}$ corresponding to $\delta=0$ shows that the bandwidth-induced 
asymmetry is small. The effect of our irreversible atomic redistribution mechanism is to contribute an additional cause for asymmetry. In particular, the ratio of the heights of the two side peaks, for $\lambda=0$, is given by

$$
\frac{\eta^{2}(1+\delta)+\delta\left(\theta^{2}-\Omega^{\prime} \theta\right)}{\eta^{2}(1+\delta)+\delta\left(\theta^{2}+\Omega^{\prime} \theta\right)}
$$

which, for example, for $\theta=\eta / 2$ yields a ratio of $(1+\delta / 2) /(1+2 \delta)$.

Asymmetries in the spectrum of resonance fluorescence under nearly monochromatic excitation have been observed experimentally. (Schuda et al., 1974; Wu et al., 1975; Hartig et al., 1976; Grove et al., 1977; Wu et al., 1977; Citron et al., 1977; Ezekiel and Wu, 1978; Cresser et al., 1982). It has been argued that they may be due to the bandwidth of the driving laser beam used in the experiments (Kimble and Mandel, 1977). Our results show that the postulated existence of an internal, atomic redistribution, relaxation mechanism may contribute an additional cause of asymmetries in the off-resonance fluorescence spectrum. In the weak-field limit $\eta \ll 1$, with off-resonance $(\theta \neq 0)$ excitation, the fluorescence is dominated by elastic scattering centered at the driving frequency (Kimble and Mandel, 1977), for monochromatic excitation. An inelastic contribution centered at the atomic transition frequency becomes important for broad bandwidth excitation. Figure 4 shows that atomic redistribution enhances and broadens the inelastic contribution.

It seems that with current high-resolution measurement techniques our present predictions and, hence, our underlying physical hypotheses, should be experimentally verifiable. At least, one could determine bounds to the value of the parameter $\delta$, and, therefore, to the value of the only unknown constant of the theory, namely, the internal redistribution inherent time constant $\tau$ appearing in equation (1).

\section{ABSORPTION AND STIMULATED EMISSION}

We now consider a two-level atom simultaneously excited by a strong, driving field with fixed spectrum centered at $\omega_{1}$ and effective bandwidth $\beta \lambda$, and by a weak, tunable monochromatic probe field of frequency $\omega$. The rate of absorption of energy from the weak probe field is proportional to the time-dependent line-shape function (Mollow, 1972; 1975)

$$
\int_{0}^{t} d \tau\left\langle\left\langle\left[\mathbf{g}_{a}(t-\tau, \tau)\right]_{1}\right\rangle e^{i \omega \tau}+\right.\text { c.c. }
$$

which in the steady state long-time limit becomes

$$
\Phi_{a}(\nu)=\int_{0}^{\infty} d \tau\left[\boldsymbol{\Gamma}_{a s}(\infty, \tau)\right]_{1} e^{i \beta \nu \tau}+\text { c.c. }
$$




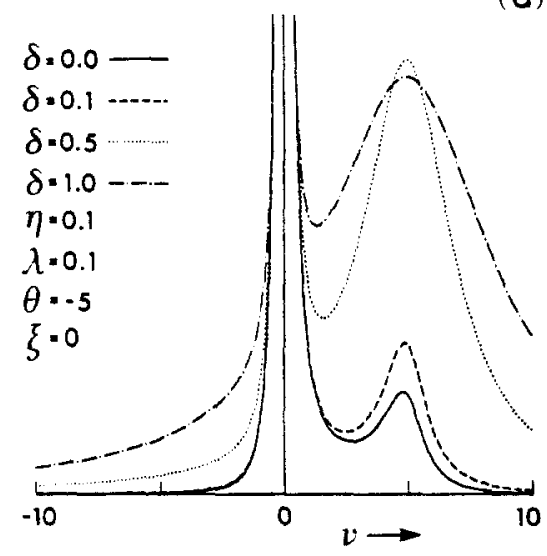

Fig. 4. Resonance fluorescence spectrum $\Phi_{e}(\nu)$ versus $\nu$. Dependence on the internal redistribution parameter $\delta$ in the weak-field limit $(\eta=0.1)$ off-resonance $(\theta=-5)$. (a) Small excitation bandwidth $(\lambda=0.1)$. (b) Large excitation bandwidth $(\lambda=1)$.

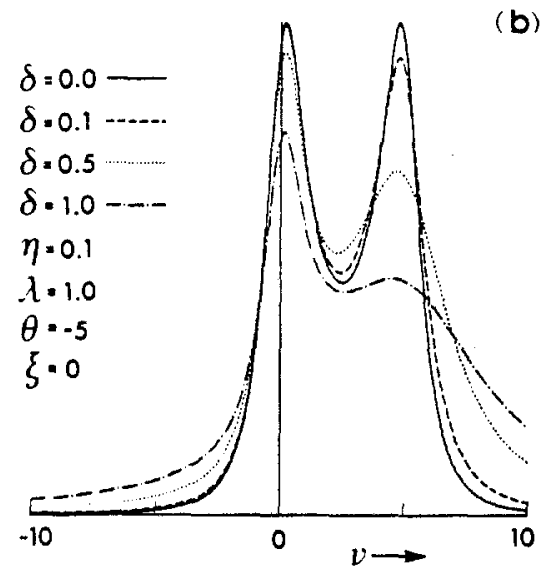

where $\nu=\left(\omega-\omega_{1}\right) / \beta$. Negative values of the absorption line-shape function $\Phi_{a}(\nu)$ represent stimulated emission, i.e., amplification of the probe field. With the help of equations (41), (43), and (46), we find

$$
\begin{array}{r}
\boldsymbol{\Gamma}_{a s}(\infty, 0)=\left|\begin{array}{c}
2\left[(\mathbf{A}+\mathbf{C})^{-1}\right]_{22} \\
-2\left[(\mathbf{A}+\mathbf{C})^{-1}\right]_{32} \\
0
\end{array}\right| \\
\beta \int_{0}^{\infty} d \tau e^{i \beta \nu \tau} \boldsymbol{\Gamma}_{a s}(\infty, \tau)=\left[(\mathbf{A}+\mathbf{D}-i \nu \mathbf{l})^{-1}\right] \cdot \boldsymbol{\Gamma}_{a s}(\infty, 0)
\end{array}
$$


and, after some manipulations, we find the following two equivalent expressions for the absorption line-shape:

$$
\begin{aligned}
\beta \Phi_{a}(\nu) & =\frac{2 c}{\eta^{2}}\left\{\frac{m(-i \nu)}{f(-i \nu)}+\text { c.c. }\right\} \\
& =\frac{4 c}{\eta^{2}} \frac{a^{\prime}(\nu) u(\nu)+b^{\prime}(\nu) v(\nu)}{u(\nu)^{2}+v(\nu)^{2}}
\end{aligned}
$$

where $c, f(p), u(\nu)$, and $v(\nu)$ are defined by Equations (59), (60), and (65), respectively, and

$$
\begin{aligned}
m(p)= & -\eta^{2}(3 \lambda+p)(1+\Delta+\lambda-i \theta) \\
& +\left[(2+\lambda+p)(1+\Delta+4 \lambda+p+i \theta)+2 i \Delta \xi \eta^{2}\right]\left[(1+\Delta+\lambda)^{2}+\theta^{2}\right]
\end{aligned}
$$
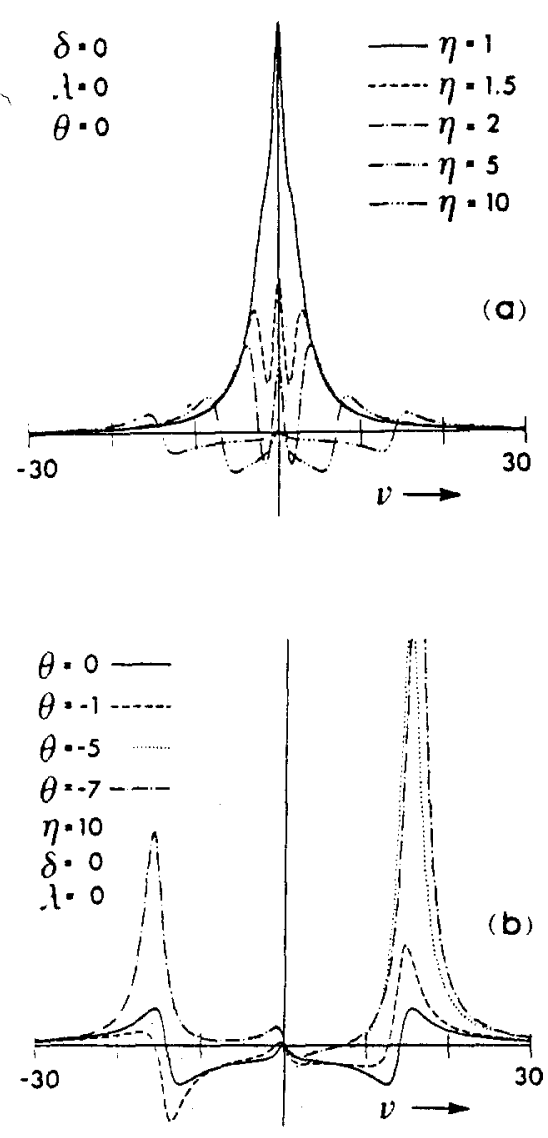

Fig. 5. Absorption and stimulated emission line shape $\Phi_{a}(\nu)$ versus dimensionless monochromatic probe-field frequency $\nu$. (a) Dependence on the driving field strength $\eta$ on resonance $(\theta=0)$ with monochromatic driving field $(\lambda=0)$, (b) Dependence on the detuning parameter $\theta$ for a strong $(\eta=10)$ monochromatic $(\lambda=0)$ driving field. 


$$
\begin{aligned}
= & {\left[(1+\Delta+\lambda)^{2}+\theta^{2}\right] p^{2}+\left\{(3+\Delta+5 \lambda+i \theta)\left[(1+\Delta+\lambda)^{2}+\theta^{2}\right]\right.} \\
& \left.-\eta^{2}(1+\Delta+\lambda-i \theta)\right\} p+[(2+\lambda)(1+\Delta+4 \lambda+i \theta) \\
& \left.+2 i \Delta \xi \eta^{2}\right]\left[(1+\Delta+\lambda)^{2}+\theta^{2}\right]-3 \eta^{2} \lambda(1+\Delta+\lambda-i \theta) \\
a^{\prime}(\nu)= & \operatorname{Re}[m(-i \nu)]=-\left[(1+\Delta+\lambda)^{2}+\theta^{2}\right]\left[\nu^{2}+\nu \theta-(2+\lambda)(1+\Delta+4 \lambda)\right] \\
& +\eta^{2}[\nu \theta-3 \lambda(1+\Delta+\lambda)] \\
b^{\prime}(\nu)= & \operatorname{Im}[m(-i \nu)]=-\nu\left[(1+\Delta+\lambda)^{2}+\theta^{2}\right](3+\Delta+5 \lambda)+\theta(2+\lambda) \\
& +\eta^{2}[\nu(1+\Delta+\lambda)+3 \lambda+2 \Delta \xi]
\end{aligned}
$$

In the absence of the postulated atomic redistribution mechanism $(\delta=0)$, Figure 5a shows the well-known absorption line shapes for different driving field strengths. In the strong-field limit $(\eta=10)$, Figure $5 \mathrm{~b}$ shows the effect of detuning.

The effect of $\delta$ on the on-resonance $(\theta=0)$ stimulated absorptionemission line shape is shown in Figure 6 for both a strong $(\eta=10)$

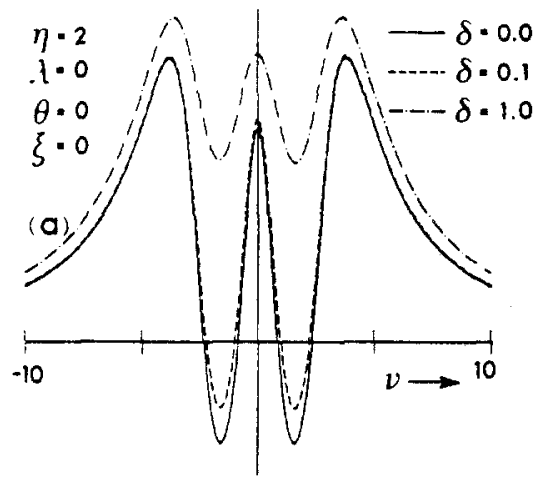

Fig. 6. Absorption and stimulated emission line shape $\Phi_{a}(\nu)$ versus $\nu$ on resonance $(\theta=$ $0)$ with monochromatic driving field $(\lambda=0)$. Dependence on the internal redistribution parameter $\delta$. (a) Driving field strength $\eta=2$. (b) Driving field strength $\eta=10$.

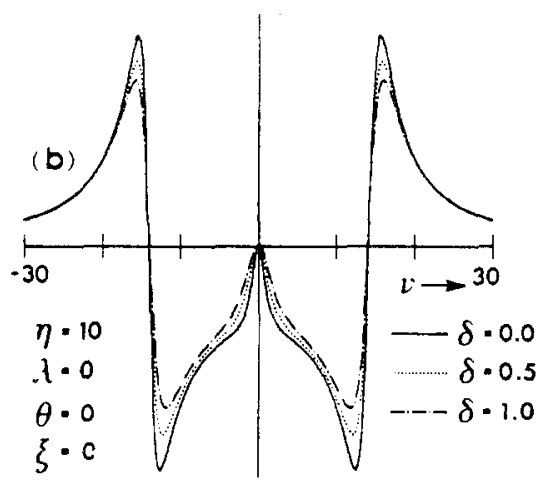



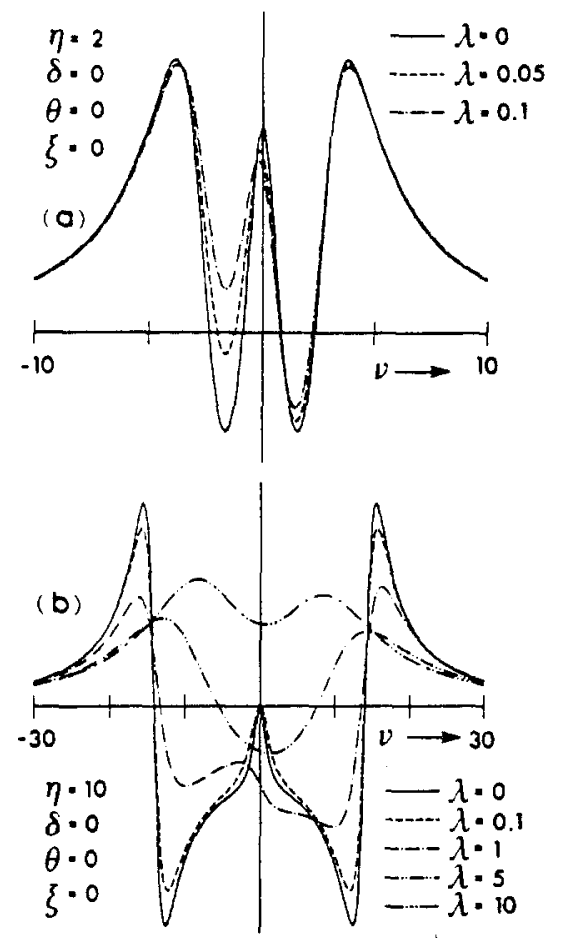

Fig. 7. Absorption and stimulated emission line shape $\Phi_{a}(\nu)$ versus $\nu$ on resonance $(\theta=$ 0 ). Effect of driving-field bandwidth $\lambda$ (redistribution parameter $\delta=0$ ). (a) Driving field strength $\eta=2$. (b) Driving field strength $\eta=$ 10.

monochromatic $(\lambda=0)$ driving field, and a weaker $(\eta=2)$ monochromatic $(\lambda=0)$ driving field, for which the relative effect is particularly appreciable (Figure 6a). It is noteworthy that a nonzero value of $\delta$ introduces no asymmetries in the line shape. Asymmetries are instead introduced by a nonzero excitation bandwidth $\lambda$ (Figure 7). Thus, in the presence of both a nonzero $\delta$ and a nonzero $\lambda$ (Figure 8 ) the contributions of the two effects are clearly distinguishable.

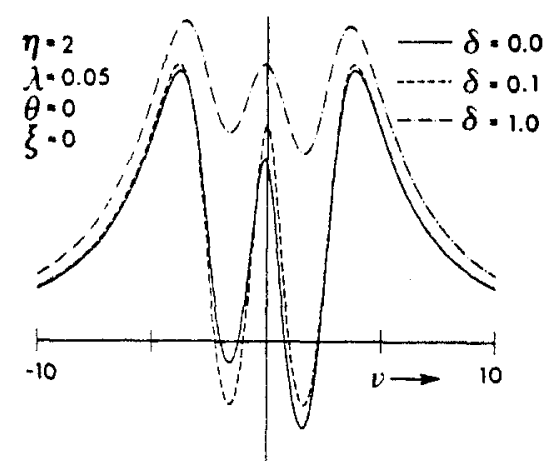

Fig. 8. Absorption and stimulated emission line shape $\Phi_{a}(\nu)$ versus $\nu$ on resonance $(\theta=$ 0 ) with driving field strength $\eta=2$ and bandwidth $\lambda=0.05$. Effect of the internal redistribution parameter $\delta$. 
Again, we submit that a quantitative, high-resolution experimental study is needed to verify our predictions and, hence, the validity of the fundamental physical hypotheses of quantum thermodynamics.

\section{CONCLUSIONS}

We have postulated the existence of an internal atomic relaxation mechanism by which nonpure individual quantum states of a single isolated uncorrelated two-level atom tend to approach a stable equilibrium state. This relaxation mechanism is entailed by a novel nonlinear equation of motion [equation (1)] proposed by the author as an attempt to resolve the long-standing dilemma on the nature of entropy and irreversibility.

We have estimated the corrections that our constant-energy irreversible atomic relaxation implies on the spectral distribution of the resonance fluorescence and the absorption and stimulated emission line shape from a two-level atom driven about resonance by a finite-bandwidth quantum electromagnetic laser field.

Verification of some of our predictions, or at least the determination of bounds to the value of the unknown internal redistribution time constant $\tau$ may be within reach of current high-resolution experimental techniques.

\section{ACKNOWLEDGMENTS}

The author is indebted with Professor Luigi A. Lugiato, Universita' di Milano, for an inspiring conversation, and has greatly benefited from the continuing personal interest and support of Dr. George N. Hatsopoulos, Thermo Electron Corporation.

\section{REFERENCES}

Ackerhalt, J. R., and Eberly, J. H. (1974). Physical Review D, 10, 3350.

Ackerhalt, J. R., Knight, P. L., and Eberly, J. H. (1973). Physical Review Letters, 30, 456.

Beretta, G. P. (1981). Ph.D. Thesis, M.I.T., unpublished.

Beretta, G. P., Gyftopoulos,:E. P., Park, J. L., and Hatsopoulos, G. N. (1984). Nuovo Cimento $B, 82,169$.

Beretta, G. P., Gyftopoulos, E. P., and Park, J. L. (1985). Nuovo Cimento B, 87, 77.

Beretta, G. P. (1985). International Journal of Theoretical Physics, 24, 119.

Beretta, G. P., in press. In Frontiers of Nonequilibrium Statistical Physics, G. T. Moore and M. O. Scully, eds. Plenum Press, New York.

Beretta, G. P. (1985). International Journal of Theoretical Physics, 24, 119.

Citron, M. L., Gray, H. R., Gabel, C. W., and Stroud, C. R., Jr. (1977). Physical Review A, $16,1507$.

Cohen-Tannoudji, C., and Reynaud, S. Journal Physics B, 10, 2311.

Cresser, J. D., Hager, J., Leuchs, G., Rateike, M., and Walther, H. (1982). In Dissipative Systems in Quantum Optics, R. Bonifacio, ed. Springer-Verlag, New York, p. 21. and references therein. 
Ezekiel, S., and Wu, F. Y. (1978). In Multiphoton Processes, J. H. Eberly and P. Lambropoulos, eds. Wiley, New York, p. 145.

Grove, R. E., Wu, F. Y., and Ezekiel, S. (1977). Physical Review A, 15, 227.

Hartig, W., Rasmussen, W., Schieder, R., and Walther, H. (1976). Zeitschrift für Physik A, 278, 205.

Hatsopoulos, G. N., and Gyftopoulos, E. P. (1976a). Foundations of Physics, 6, 15.

Hatsopoulos, G. N., and Gyftopoulos, E. P. (1976b). Foundations of Physics, 6, 127.

Hatsopoulos, G. N., and Gyftopoulos, E. P. (1976c). Foundations of Physics, 6, 439.

Hatsopoulos, G. N., and Gyftopoulos, E. P. (1976d). Foundations of Physics, 6, 561.

Kimble, H. J., and Mandel, L. (1975). Physical Review A, 13, 2123.

Kimble, H. J., and Mandel, L. (1977). Physical Review A, 15, 689.

Mollow, B. R. (1969). Physical Review, 188, 1969.

Mollow, B. R. (1972). Physical Review A, 5, 2217.

Mollow, B. R. (1975). Physical Review A, 12, 1919.

Mollow, B. R. (1981). In Progress in Optics, Vol. XIX, E. Wolf, ed. North-Holland, New York, p. 3.

Park, J. L., and Simmons, R. F. Jr. (1983). In Essays in Honor of Wolfgang Jourgrau, A. van der Merwe, ed. Plenum Press, New York.

Peuse, B. W., Prentis, M. G., and Ezekiel, S. (1982). Physical Review Letters, 49, 269.

Schuda, F., Stroud, C. R., Jr. and Hercher, M. (1974). Journal of Physics B, 7, L189.

Wu, F. Y., Grove, R. E., and Ezekiel, S. (1975). Physical Review Letters, 35, 1426.

Wu, F. Y., Ezekiel, S., Ducloy, M., and Mollow, B. R. (1977). Physical Review Letters, 38, 1077. 